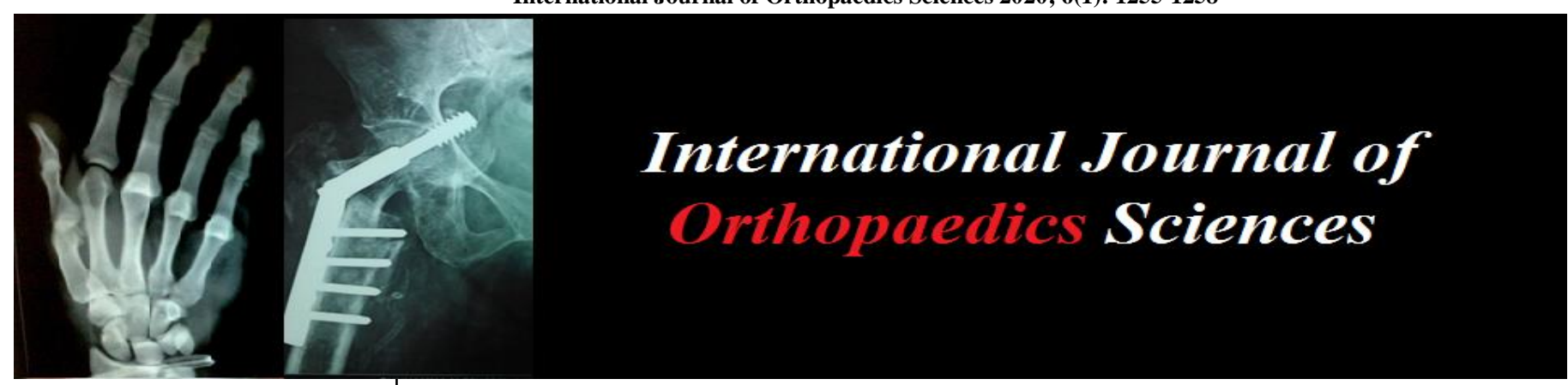

E-ISSN: 2395-1958

P-ISSN: 2706-6630

IJOS 2020; 6(1): 1255-1258

(C) 2020 IJOS

www.orthopaper.com

Received: 08-11-2019

Accepted: 12-12-2019

Dr. Kalyan Deepak Sreenivas Assistant Professor, Department of Orthopedics, SMVMCH, Pondicherry, India

Dr. Justin Moses C

Associate Professor, Department of Orthopedics, SMVMCH,

Pondicherry, India

Dr. Khisan Kumar PN Resident, Department of Orthopedics, SMVMCH,

Pondicherry, India
Corresponding Author: Dr. Justin Moses C

Associate Professor, Department of Orthopedics, SMVMCH,

Pondicherry, India

\section{Functional outcome of distal tibial fractures treated by stainless steel locking plates with minimally invasive plate Osteosynthesis in rural population: A prospective study}

\author{
Dr. Kalyan Deepak Sreenivas, Dr. Justin Moses C and Dr. Khisan Kumar PN
}

DOI: https://doi.org/10.22271/ortho.2020.v6.i1q.1990

Abstract

Introduction: Open reduction and plating of distal tibial fractures require extensive soft tissue dissection and periosteal stripping. Complications like infection, delayed union and non-union are common. Minimally invasive plate Osteosynthesis (MIPO) technique preserves soft tissue envelope, vascularity of fracture fragments and containment of fracture haematoma and provide biologically favourable environment for fracture healing.

Materials and methods: 20 patients of distal tibial fractures treated with locking plate by MIPO technique between October 2015 and January 2017 were included in this prospective study. Analysed using software SPSS version 24.0

Results: Mean average time to union is 15.2 weeks. Based on Tenny and Wiss scoring criteria, eighteen patients $(90 \%)$ had good and excellent results at 22 weeks with fracture union.

Conclusion: MIPO technique provides good healing and decreases the incidence of non-union and the need for bone grafting. This technique can be used in distal tibia fractures where nailing cannot be done, such as, fractures with small distal metaphyseal fragments, coronal fractures, Comminuted and intraarticular fractures.

Keywords: stainless steel locking plates, minimally invasive plate Osteosynthesis

\section{Introduction}

The distal part of the tibia is superficial with less soft tissue coverage. Injuries to this region cause more morbidity due to compromised blood supply, soft tissue damage and involvement of the articular surface. With the increase in high velocity trauma resulting in more complex fracture patterns, the management of distal tibial fractures has become more challenging. Fracture pattern, soft tissue injury and bone quality influence the selection of fixation technique ${ }^{[1]}$. External fixators can lead to inadequate reduction, malunion, non-union and pin tract infection ${ }^{[2]}$. Intramedullary nailing is not feasible because of short metaphyseal segment possibility of rotational and secondary malalignment ${ }^{[3]}$. Open reduction and plating needs extensive soft tissue dissection and periosteal stripping. Incidence of infection, delayed-union, and non-union is higher ${ }^{[4]}$. Minimally invasive plate Osteosynthesis (MIPO) technique offers biologic fixation with preservation of soft tissue, fracture haematoma and bone vascularity [5]

Threaded heads of locking screws lock into the threads of the locking plate creating a fixed angle construct which is less prone to loosening. Anatomically contoured locking plate prevents malreduction and allows a better distribution of angular and axial load. Locking plate indirectly reduces and bridges the fracture without producing compressive forces on the bone [6]. They have locking screw holes to fit cortical locking screws in the proximal and cancellous locking screws in the distal part of the plate to allow fixation of cortical and cancellous parts of tibia respectively. Separate plates are available for placement in the medial or lateral, left or right sides of the distal tibia in various shapes and sizes. The number of distal cancellous screw holes may vary with different manufacturers. The cortical screw holes are vertically oriented and the cancellous screw holes are horizontally oriented to increase the number of screw purchases ${ }^{[7]}$. 
Drill guides control the drilling angle which controls the angle of the screws in the plate. Many locking screw holes are accompanied by non-locking screw holes to facilitate the use of non-locking cortical and cancellous screws to provide compression before fixation with locking screws. They are available in both $3.5 \mathrm{~mm}$ and $4.5 \mathrm{~mm}$ systems. The objective of this study is to analyse the outcomes of MIPO in distal tibial fractures.

\section{Materials and methods}

2.1 study design: This study was done at Sri Manakula Vinayagar Medical College and Hospital in Puducherry after obtaining institutional ethical committee approval. It is a prospective study of patients with distal tibial fractures who attended the institute between October 2015 and January 2017. A total of 20 patients who met the criteria were analysed.

\subsection{Inclusion criteria}

1. Closed distal tibial fractures

2. Patients with age 18 years and above.

3. Gustilo- Anderson Grade 1 \& 2 distal tibial fractures.

4. Pilon fractures

\subsection{Exclusion criteria}

1. Patients with age $<18$ years

2. Gustilo- Anderson Grade 3 distal tibial fractures

3. Patients with pathological distal tibial fractures other than osteoporosis

4. Distal tibial fractures with neurovascular deficit.

\subsection{Procedure}

After resuscitation, all patients were evaluated with anteroposterior and lateral x-rays of ankle joint and leg. CT was done in Articular fractures. MRI of ankle joint was reserved for patients with ligament injuries. All fractures were classified as per AO classification.

After obtaining anaesthetic fitness, fracture fixation using LCP by MIPO technique was performed. Prophylactic intravenous antibiotics were administered before induction of anaesthesia

Under epidural/spinal anaesthesia, patient was placed in a supine position on a radiolucent table. After preparation of parts, a $4 \mathrm{~cm}$ incision was made over medial malleolus avoiding saphenous vein and nerve. Under the guidance of image intensifier, fracture reduction was done. Plate was inserted over the periosteum and fixed with $\mathrm{K}$ wires to maintain reduction. Stab incisions were made over the holes using identical plate or under $\mathrm{C}$ arm control. Using locking sleeves, the screw holes were drilled and appropriate screws were inserted. Wound washed and closed in layers.

Patients were allowed ankle and toe mobilization immediately after the pain subsided. Limb elevation was maintained. Non weight bearing crutch walking was started. Below knee slab was applied if the patient had associated injuries. Partial weight bearing was started after 1 month. Patients were assessed clinically and radiologically every 3 weeks for improvement in range of motion and reduction in pain and progression of the union and implant condition. All patients were assessed according to Tenny and Wiss scoring system [8].

\subsection{Statistical analysis}

Data was entered using the software Epi info version 7.2.1.0 and analysed using software SPSS version 24.0. categorical study variables like gender, age group, side of fracture, mode of injury, post-operative shortening, functional outcome, postoperative complications was described in terms of frequency and percentage. Continuous variables like age, operating time, duration of stay in hospital were described in mean and standard deviation. Association between age, gender and duration of immobilization with functional outcome were assessed using fisher exact probability test.

\section{Results}

Most $(40 \%)$ of the study participants were in the age group of 31-40 years. Mean age was 44.5 years. Male to female ratio was 4:1. Right side was more commonly involved (70\%) when compared to left side (30\%). Eighty five percent of the fractures united within 16 weeks of surgery whereas for 3 patients $(15 \%)$ fracture united at 18 weeks. Average time to union is 15.2 weeks. Eighteen $(90 \%)$ patients had satisfactory ankle movements leading to normal walking or a minimal limp. Two (10\%) patients had ankle stiffness and limping at 22 weeks which improved after physiotherapy. 13 patients $(65 \%)$ had more than 30 degrees of plantar flexion whereas remaining 7 patients had 11 to 30 degrees of plantar flexion at the time of final follow up. 11 patients $(55 \%)$ had 11 to 15 degrees of dorsiflexion and 7 patients $(35 \%)$ had more than 15 degrees of dorsiflexion whereas 2 patients $(10 \%)$ had less than 11 degrees of dorsiflexion. Functional outcome was assessed by Tenny and Wiss criteria. Eighteen patients (90\%) had good and excellent results. Fair results were observed in two patients. Implant removal was done at 18 months for two patients who had pain due to implant prominence. Two patients had ankle stiffness. Patient who were younger had better treatment outcomes as compared to older patients. However this association was not statistically significant ( $p$ value 0.142 ). Male patients had a better outcome as compared to female patients and this association was not statistically significant ( $\mathrm{p}$ value 0.245 ).

\section{Discussion}

Collinge and Sanders [9] have described indirect fracture reduction and percutaneous plating techniques for the lower extremity as an evolutionary step in biological plating. Redfern et al. ${ }^{[10]}$ and Borg et al. ${ }^{[11]}$ reported good results for MIPO, using closed indirect reduction and contoured dynamic compression plates for distal tibial fractures. Our study included similar numbers of patients however we have focussed on fracture healing and complications encountered with regard to our minimally invasive fixation technique.

The management of distal tibia fractures can be challenging because of the lack of adequate soft tissue coverage, subcutaneous bone, and poor vascularity. The surgical treatment of a distal tibial fracture must achieve the necessary mechanical stability without impairing callus formation. The distal tibia is covered only by a very thin layer of soft tissue, so that the treatment of distal tibial fractures is demanding, and complications occur frequently.

The timing of surgery should be optimized to allow the oedema to subside and soft tissues to heal which help to minimise the postoperative wound related problems. ${ }^{12}$ Locking plates have a better holding power because of fixed angular stability through the head of locking screws. Minimally invasive plating techniques reduce iatrogenic soft tissue injury and damage to bone vascularity, in addition to preserving the osteogenic fracture hematoma ${ }^{[13]}$.

Primary stabilisation with external fixation is much safer where soft tissue is extensively damaged. Concomitant fibular 
fractures should be stabilised with a plate as early as possible, thereby increasing the stability of the tibial reduction, facilitating the correct alignment of the tibia, and preventing a loss of the limb length ${ }^{[14]}$. An external fixator can be used for the definitive treatment, and can be combined with cancellous bone graft in cases of delayed healing. If the fracture does not heal completely, mobilisation is done in a cast after removal of the fixator. But there are disadvantages. Fracture healing takes a longer time especially if there is significant soft-tissue damage ${ }^{[15]}$. The stability of the external fixator is not sufficient for early full weight-bearing, and the compression on the fracture is very limited. Bridging plate Osteosynthesis permits micro motion between the individual fragments, whereby the healing process is stimulated by callus formation. The implant selected must be of adequate length to achieve bridging of the fracture site.

In periarticular fractures, additional incisions may be needed to visualize and assess the joint surface to ensure that the articular surfaces are anatomically aligned. The technically demanding procedure and the increased exposure to radiation have to be considered.

\section{Case 1}

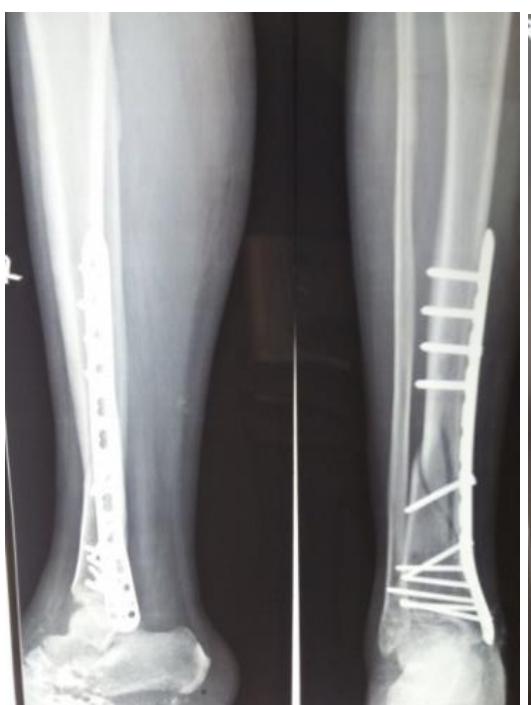

Pre op

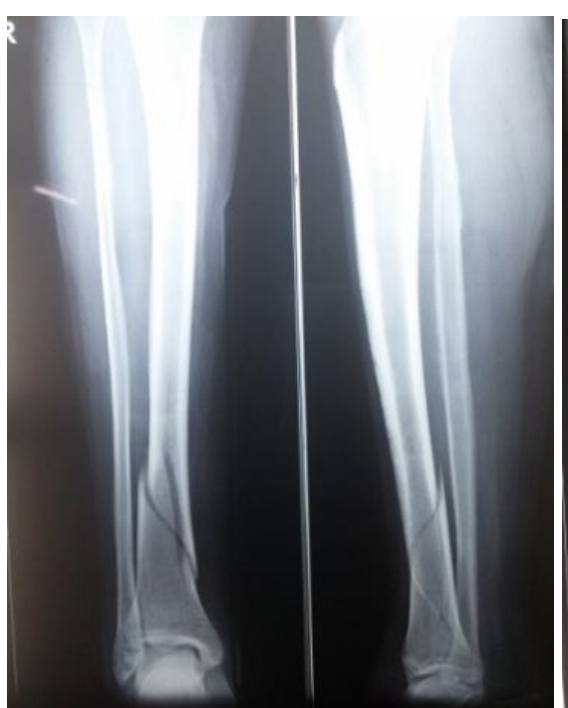

Immediate post op

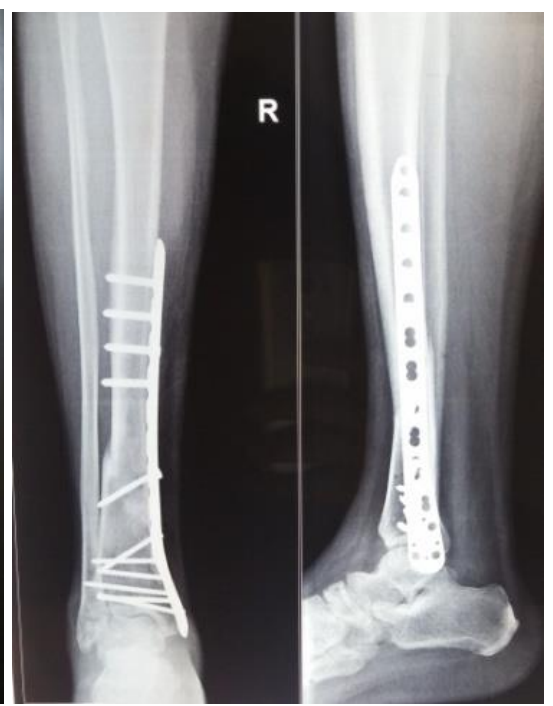

6- month post op

Case 2

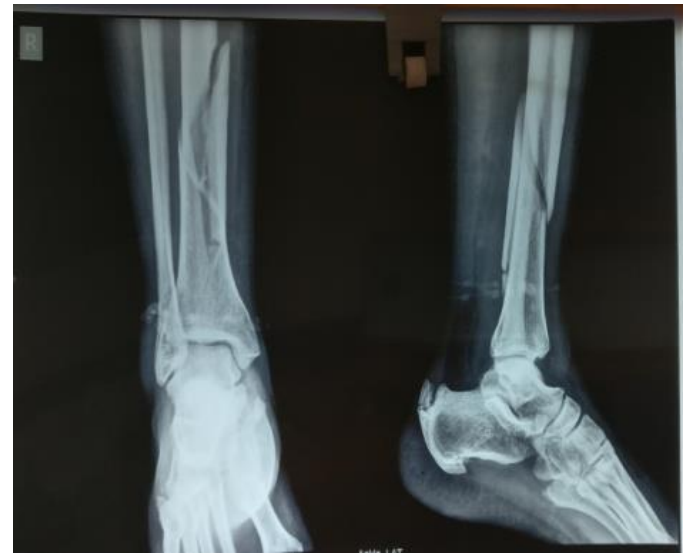

Pre op

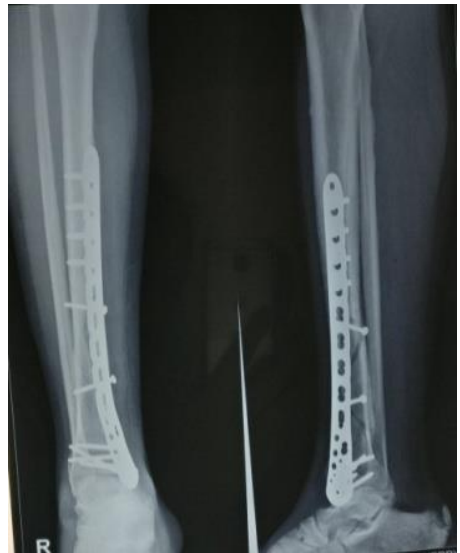

Immediate post op

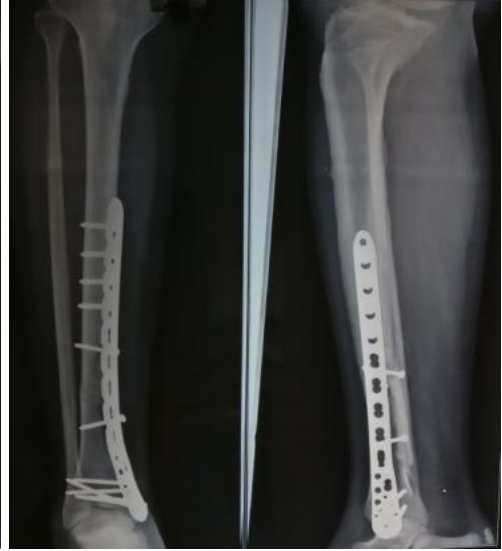

6- month post op

\section{Conclusion}

Minimally invasive locking plate Osteosynthesis aims to reduce surgical tissue trauma and help in preserving the periosteal vascular integrity and osteogenic fracture haematoma. Based on our study, good and excellent results were obtained in $90 \%$ of the cases. While fair results were noted in two patients. Ankle stiffness was attributed to old age and associated injuries which improved with physiotherapy. More randomised controlled studies on MIPO versus other modes of internal fixation are required. We conclude that the use of locking compression plate for distal tibial fractures through minimally invasive technique is safe and effective.

\section{Reference}

1. Bedi A, Le TT, Karunakar MA. Surgical treatment of non-articular distal tibia fractures. J Am Acad. Orthop Surg. 2006; 14(7):406-16.

2. Rammelt S, Endres T, Grass R et al. The role of external fixation in acute ankle trauma. Foot Ankle Clin. 2004; 9(3):455-74.

3. Boenisch UW, de Boer PG, Journeaux SF. Unreamed intramedullary tibial nailing-fatigue of locking bolts. Injury. 1996; 27(4):265-70.

4. Fisher WD, Hamblen DL. Problems and pitfalls of compression fixation of long bones fractures: a review of results and complications. Injury. 1978; 10(2):99-107. 
5. Mushtaq A, Shahid R, Asif M et al. Distal Tibial Fracture Fixation with Locking Compression Plate (LCP) Using the Minimally Invasive Percutaneous Osteosynthesis (MIPO) Technique. Eur. J Trauma Emerg Surg. 2009; 35:159-164.

6. Cantu RV, Koval KJ. The use of locking plates in fracture care. J Am Acad. Orthop Surg. 2006; 14(3):18390.

7. Frigg R. Development of locking compression plate. Injury. 2003; 34(2):B6-10.

8. Tenny SM, Wiss DA. Open reduction and internal fixation of tibial plafond fractures. Clin Orthop 1993; 292:108.

9. Collinge CA, Sanders RW. Percutaneous plating in the lower extremity. J Am Acad. Orthop Surg. 2000; 8(4):211-6.

10. Redfern DJ, Syed SU, Davies SJM. Fractures of the distal tibia. Injury. 2004; 35:615-20.

11. Borg T, Larsson, Lindsjo U. Percutaneous plating of distal tibial fractures. Preliminary results in 21 patients. Injury 2004; 35:608-14.

12. Shah BM, Somshekhar PN. MIPO with anatomical plate for distal tibia extra articular fractures: A safe and effective modality. International Journal of Orthopaedics. 2019; 5(1):12-7.

13. Gupta P, Tiwari A, Thora A, Gandhi JK, Jog VP. Minimally invasive plate Osteosynthesis (MIPO) for proximal and distal fractures of the tibia: A biological approach. Malaysian orthopaedic journal. 2016; 10(1):29.

14. Vidović D, Matejčić A, Ivica M, Jurišić D, Elabjer E, Bakota B. Minimally-invasive plate Osteosynthesis in distal tibial fractures: results and complications. Injury. 2015; 46:S96-9.

15. Babis GC, Kontovazenitis P, Evangelopoulos DS, Tsailas P, Nikolopoulos K, Soucacos PN. Distal tibial fractures treated with hybrid external fixation. Injury. 2010; 41(3):253-8. 